\title{
Strangeness and Charm Production in High Energy Heavy Ion Collisions
}

\author{
N. $\mathbf{X u} \S$ \\ Lawrence Berkeley National Laboratory, Berkeley, CA 94720, USA
}

\begin{abstract}
We discuss the dynamical effects of strangeness and charm production in high energy nuclear collisions. In order to understand the early stage dynamical evolution, it is necessary to study the transverse momentum distributions of multi-strange hadrons like $\Xi$ and $\Omega$ and charm messons like $J / \Psi$ as a function of collision centrality.
\end{abstract}

\section{Introduction}

About 20 years ago, when we began the heavy ion collisions program, strangeness production was established to be a useful tool for understanding the collision dynamics. The 'standard' suggestion is that the enhancement of the strangeness production is a signal for the formation of quark-gluon plasma [1]. On the other hand it was argued that "in the central region, strangeness is not a signal of the existence of a quarkgluon plasma, although an enhanced strangeness production might signal interesting dynamical phenomena." [2]. Today, with the collection of vast amounts of data, from collisions ranging from $\sqrt{s_{\mathrm{NN}}} \sim 1$ to $200 \mathrm{GeV}$, we are approaching a much better understand of the strangeness world: (1) Strangeness production is strongly affected by the baryons; (2) The enhancement is due to rescatterings and has been observed in $\mathrm{p}+\mathrm{A}$ collisions [3]; (3) Transverse momentum distributions of strange particles, especially multi-strange baryons, differ from others, they freeze-out earlier.[4]

To illustrate the above points, we will focus on recent measurements of kaon over pion ratios, thermal fits to particle yield ratios, and particle transverse properties. Systematics as a function of collision energy will be our guidence throughout the paper. In addition to the strangeness, we also include the subject of charm production in high energy nuclear collisions.

\section{Experimental results}

\subsection{Kaon over pion ratio systematics}

Kaons, as the lightest strange mesons, carry most of the strangeness produced in heavy ion collisions. One of the traditional ways to compare the $s$-quark production to that of the light quarks $u$ (or $d$ ) is the ratio of kaon over pion yields. The systematics of mid-rapidity kaon over pion ratios [5] as a function of center of mass energy are shown in Figure 1 (a) and (b). In Fig. 1(a), the positive $k / \pi$ ratio shows a peak 
at about $\sqrt{s_{\mathrm{NN}}}=8 \mathrm{GeV}$. After the peak, the ratio continously decrases all the way to RHIC energies. At midrapidity, the highest possible baryon density peaks at the beam energy of about $8 \mathrm{GeV}$ as indicated by the positive kaon over pion ratio. This behavior clearly reflects the dynamics of baryons. On the other hand, the negative $k / \pi$ ratio shows a monotopic rise from AGS to SPS to RHIC indicating that the strange quark pair production becomes more important at higher beam energy. These features were predicted by transport model calculations [6, 7] and the general trend was also well produced by thermal model calculations [8]. The dominant factor here is the baryon distribution as a function of beam energy. This non-trival observation is, of course, related to issues of baryon transport and strange baryon pair production in high energy nuclear collisions [9]. At the peak energy, the freeze-out parameters already reached the plateau region [10].

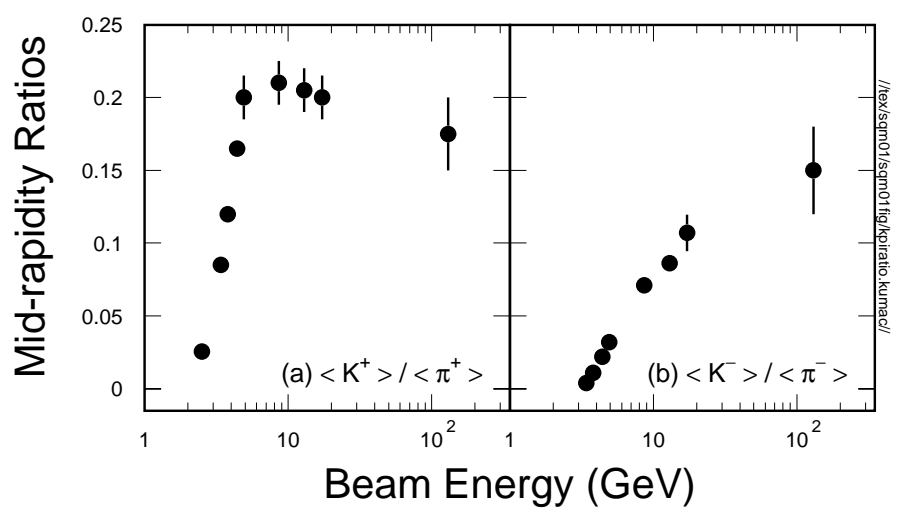

Figure 1. Mid-rapidity negative kaon over pion ratio as a function of center of mass energy.

\subsection{Thermal model fits}

Statistical models $[11,12,13,14,15]$ have been applied to the results of particle yield ratios from both elementary collisions $\left(e+e^{-}, p+p\right)$ and heavy ion collisions $(A u+A u$ and $P b+P b)[11]$. Many features of the data imply that a large degree of chemical equilibration has been reached in collisions at the AGS, SPS, and RHIC. The three most important results are: (i) at high energy collisions the chemical freezeout (inelastic collisions cease) occurs at about $160-180 \mathrm{MeV}$ and it is 'universal' to both elementary and heavy ion collisions; (ii) the kinetic freeze-out (elastic scatterings cease) occurs at a lower temperature $\sim 120-140 \mathrm{MeV}$ for copiously produced particles; (iii) the compilation of freeze-out parameters [13] in heavy ion collisions in the energy range from 1 - $200 \mathrm{~A} \cdot \mathrm{GeV}$ shows that a constant energy per particle $\langle E\rangle /\langle N\rangle \sim 1$ $\mathrm{GeV}$ can reproduce the behavior in the temperature-potential $\left(T_{c h}-\mu_{B}\right)$ plane [13]. It is worth noting that the relative enhancement of the multi-strange baryons can be well fitted if strangeness conservation is formulated in a canonical ensemble [16]. Fluctuations [17] were found to play an important role in driving the system to approach equilibrium.

The meaning of the success of the thermal models fits is yet to be understood. Whether the system under study approaches the equilibrium by phase-space filling or via interactions among particles is unknown. However, due to the 'universal' feature 
found in both elementary and heavy ion collisions and the dynamical nature of the high energy collisions, the phase-space filling is the most plausible choice. By definition, the thermal model fitting results can not provide any dynamical information about the collision before the freeze-out.

\subsection{Transverse momentum distributions}

Measured transverse momentum distributions have been fitted by the exponential function $f=A \cdot \exp \left(-m_{t} / T\right)$, where $T$ is the slope parameter and $A$ is the normalization constant. The magnitude of the slope parameter provides information on temperature (random motion in local rest frame) and collective transverse flow. Figure 2 shows the measured particle slope parameters from $\mathrm{Pb}+\mathrm{Pb}$ central collisions at SPS $\left(\sqrt{s_{\mathrm{NN}}}=17.2 \mathrm{GeV}\right)$ energies $[18,19,20,21,22]$. For central collisions the $\phi$ slope parameter of NA49 is about $300 \mathrm{MeV}$ [23] whereas that of NA50 is about 240 $\mathrm{MeV}$. The systematics of the transverse momentum distributions for $J / \Psi$ was reported by the NA50 experiment $[24,25]$. It is interesting to note that the slope parameter of $J / \Psi$ is similar to those for $\phi$ and $\Omega$.

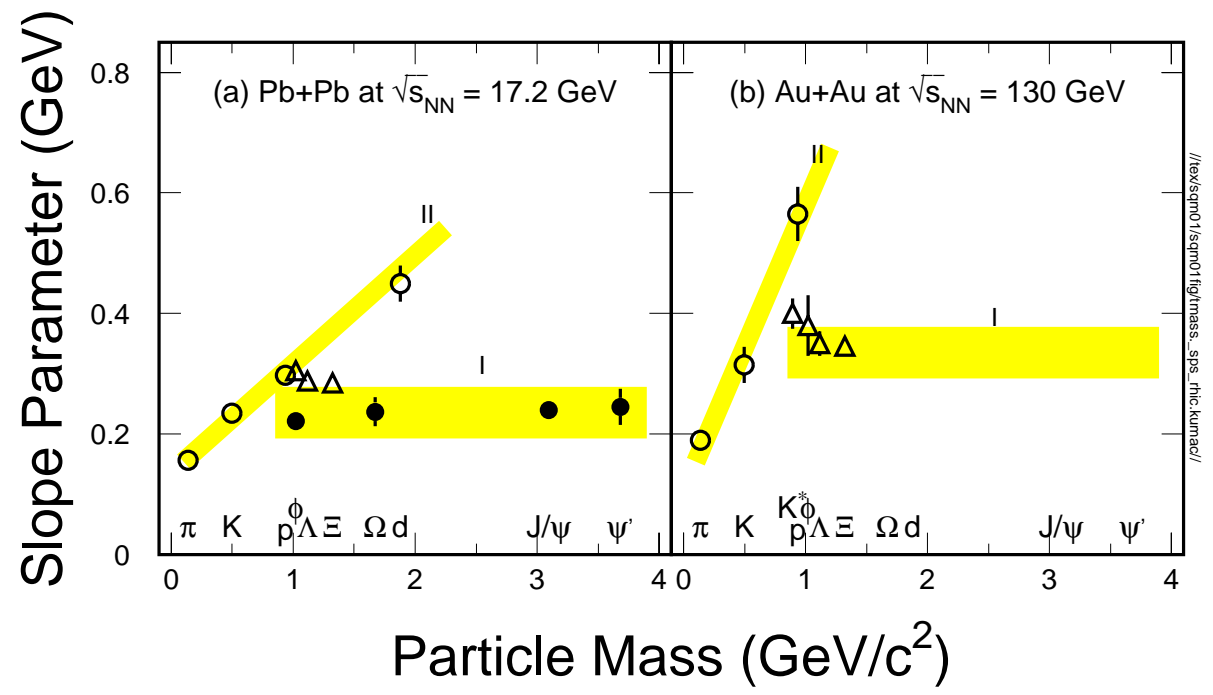

Figure 2. Slope parameter as a function of particle mass. Only data from central $\mathrm{Pb}+\mathrm{Pb}$ collisions at $\sqrt{s_{\mathrm{NN}}}=17.2 \mathrm{GeV}$ were used.

As one can see from Fig. 2(a), the slope parameters appear to fall into two groups: group (I) is flat as a function of particle mass, whereas the slopes in group (II) increase strongly with particle mass. At RHIC, the slope parameter systematic of $\pi, K$, and $p$ shows an even stronger dependence on the particle mass. The strong energy dependence of the slope parameter might be the result of the larger pressure gradient at the RHIC energy $\|$. Note that the measured values for kaons, $\Lambda$ and $\Xi$ at the RHIC energy are all higher than that from collisions at the SPS energy. With a set of reasonable initial and freeze-out conditions and equation of state, the stronger

I| A word of caution is called for here: the anti-proton slope parameter was extracted from a relatively low $p_{t}$ measurement compared to $\phi, \Lambda$ and $\Xi$. In case of strong flow the resulting slope parameter is biased. 
transverse expansion at RHIC was, in deed, predicted by hydrodynamic calculations $[26,27]$.

In a hadronic gas, the interaction cross sections for particles like $\phi, \Omega$, and $J / \Psi$ are smaller than that of $\pi, K$, and $p$ [4]. Therefore the interactions between them and the rest of the system are weak, leading to the flat band behavior in Fig. 2(a). On the other hand, the slope parameter of these weakly interacting particles may reflect some characteristics of the system at hadronization. Then it should be sensitive to the strength of the color field [28, 29, 30]. Under this assumption, the fact that the weak interacting particles show a flat slope parameter as a function of their mass would indicate that transverse flow develops at a later stage of the collision.

The systematic of the slope parameter as a function of collision centrality for $\phi$, $\Omega$, and $J / \Psi$ from $\mathrm{Au}+\mathrm{Au}$ collisions at $\sqrt{s_{\mathrm{NN}}}=17.2 \mathrm{GeV}$ is shown in Figure 3 . NA49 (open squares) and NA50 (open circle) reconstructed $\phi$ mesons via $K^{+} K^{-}$and $\mu^{+} \mu^{-}$ channels, respectively. As discussed in refs. [31, 32, 33], part of the difference may be caused by the final state interaction of the decay kaons. For peripheral collisions the slope parameters from both decay channels agree and the value is close to that from $p+p$ collisions $[23,24]$. The centrality dependence of the $\phi\left(\phi \rightarrow K^{+} K^{-}\right)$slope parameters from $\mathrm{Au}+\mathrm{Au}$ at $\sqrt{s_{\mathrm{NN}}} \sim 5 \mathrm{GeV}$ was also reported [34].

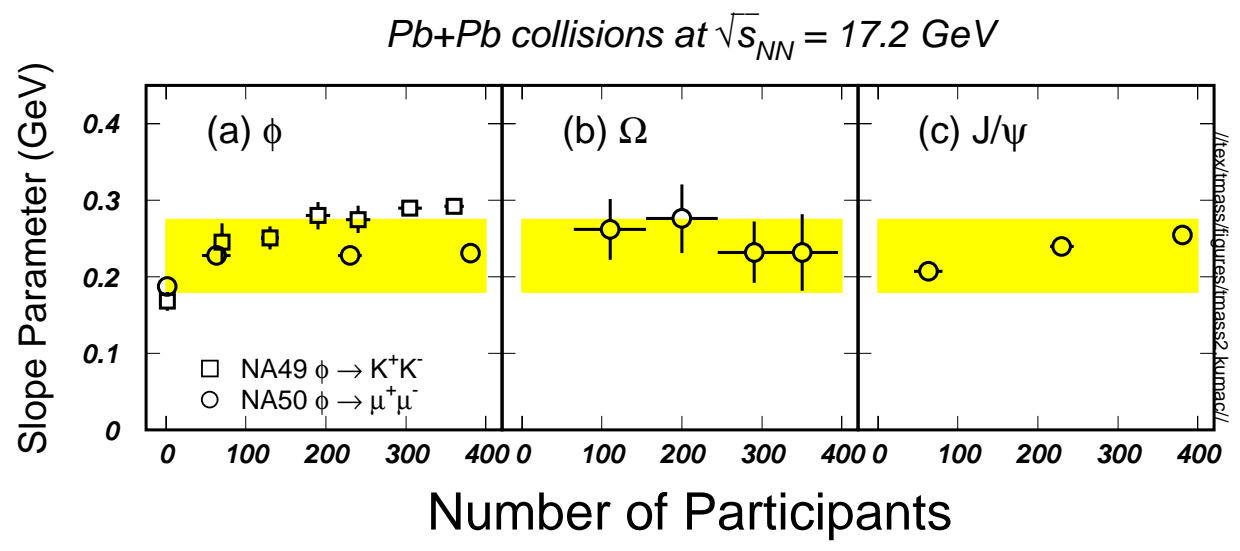

Figure 3. Slope parameter as a function of collision centrality for $\phi, \Omega$, and $J / \Psi$ from $\mathrm{Au}+\mathrm{Au}$ collisions at $\sqrt{s_{\mathrm{NN}}}=17.2 \mathrm{GeV}$.

At SPS, the $J / \Psi$ mean transverse motion increases as a function of the collision centrality, see Fig. 3. Several studies show that it is most likely due to initial scattering $[35,36,37]$. The production of the $J / \Psi$ reqiures hard(semi-hard) process and initial scattering plays an important role in generating particle transverse momentum at the SPS energy.

According to the Schwinger mechanism [28] the production probability for a pair of mass $m_{i}$ particles is given as:

$$
\rho_{i} \propto \exp \left[-\pi \cdot \frac{m_{i}^{2}+p_{t}^{2}}{\kappa}\right],
$$

where $\kappa$ measures the strength of the color field. Note that within the framework of the model, production of particle pairs and their transverse momenta are determined by the parameter $\kappa$. In elementary collisons, $\kappa$ simply reflects the string tension [38] with a typical value of $1 \mathrm{GeV} / \mathrm{fm}$. In high energy nuclear collisions, however, 
collectivity developed at the partonic stage would increase the value of $\kappa$. The idea was first implemented in transport models (RQMD) as 'color rope' by H. Sorge [7]. There the effective increase in the color field is modeled as coherent interactions of strings: $\kappa=\kappa_{0} \sqrt{n}$ with $\kappa_{0}=1 \mathrm{GeV} / \mathrm{c}$ and $n$ is the number of the overlapped strings. As a consequence one would expect to see a mass dependence of the slope parameters for particles like $\Omega, D$, and $J / \Psi$, if the flow developes at the partonic stage $[39,40]$. Therefore, the measurements of the $\Omega, D, J / \Psi$, and $\Psi^{\prime}$ transverse momentum distributios and their depence on collision centrality are crucial in order to see partonic collective flow at RHIC.

\subsection{Strange particle azimuthal anisotropy}

It has been argued $[41,42,43,44,45,46]$ that the parameter $v_{2}$ reflects the particle interactions at the early stage of high energy nuclear collisions. It measures the degree of transformation from the initial space-anisotropy to final momentum-anisotropy and should be sensitive to the parton density. Due to different interaction cross sections in hadronic gas, the study of the azimuthal anisotropy for strange particles is particularly interesting [47]. At this conference, the STAR preliminary results [48] on $v_{2}=\langle\cos (2 \phi)\rangle$ of $K_{S}^{0}$ and $\Lambda$ was reported.

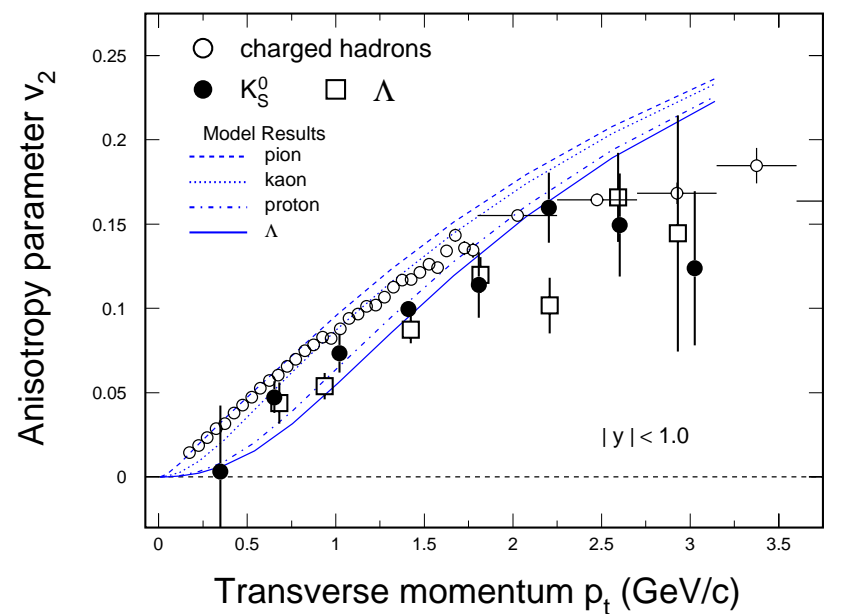

Figure 4. Azimuthal anisotropy parameters $v_{2}$ as a function of $p_{t}$ for strange particles $K_{S}^{0}$ (filled circle) and $\Lambda$ (open squares) from minimum bias $\mathrm{Au}+\mathrm{Au}$ collisions. For comparison, $v_{2}$ of pions (open circles) are also shown. Dashed-lines are from hydrodynamic model calculations. From top to bottom, respectively, are results for pions, kaons, protons, and $\Lambda$.

Shown in Figure 4 are the azimuthal anisotropy parameters $v_{2}$ as a function of $p_{t}$ for strange particles $K_{S}^{0}$ (filled circle) and $\Lambda$ (open squares) from minimum bias $\mathrm{Au}+\mathrm{Au}$ collisions a $\sqrt{s_{\mathrm{NN}}}=130 \mathrm{GeV}$. Dashed-lines represent hydrodynamic model calculations [50]. Also shown in the figure is the charged pion $v_{2}\left(p_{t}\right)$ [49]. We observe that $v_{2}$ for both strange particles increases as a function of $p_{t}$ up to about $1.5 \mathrm{GeV} / \mathrm{c}$, similar to the hydrodynamic model prediction. In the higher $p_{t}$ region $\left(p_{t} \geq 2 \mathrm{GeV} / \mathrm{c}\right)$, however, the values of $v_{2}$ seem to be saturated and smaller than the hydrodynamic predictions. This is also seen in the charged hadron (open circles) $v_{2}$. It has been 
suggested [44] that this saturation is related to the value of the parton density and the energy loss phenomena at the early stage of the evolution.

\section{Summary}

We wish to point out that, for high energy nuclear collisions, a systematic measurements of transvese momentum distributions and azimuthal anisotropy $v_{2}$ as a function of collision centrality for muti-strange hadrons and charmornium is crucial for the partonic dynamics.

\section{Acknowledgments}

The author thanks the organizers for the opportunity to discuss physics at the conference. The authors wish to acknowledge interesting discussion with Drs. M. Bleicher, M. Gorenstein, V. Koch, K. Redlich, H.G. Ritter, and S. Soff. This work was supported by the Director, Office of Energy Research, Office of High Energy and Nuclear Physics, Division of Nuclear Physics of the U.S. Department of Energy under Contract No. DE-AC0376SF00098.

\section{References}

[1] B. Müller, Nucl. Phys. A461, 213c(1987).

[2] L. McLerran, Nucl. Phys. A461, 245c(1987).

[3] B. Cole, (E910 Collaboration), Nucl. Phys. A661, 366(1999).

[4] H. van Hecke, H. Sorge, and N. Xu, Phys. Rev. Lett. 81, 5764(1998).

[5] K. Kadija, et al., (NA49 Collaboration), these proceedings.

[6] F. Wang, H. Sorge, and N. Xu, Phys. Rev. C61, 064904(2000).

[7] H. Sorge, Phys. Rev. C55, (1995).

[8] P. Braun-Munzinger, J. Cleymans, H. Oeschler and K. Redlich, LANL Preprint, hepph/0106066, June, 2001.

[9] S. Soff and N. Xu, in preparation, 2001.

[10] N. Xu et al., (NA44 Collaboration), Nucl. Phys. A610, 175c(1996).

[11] P. Braun-Munzinger, J. Stachel, J. Wessels, and N. Xu, Phys. Lett. B344, 43(1995); Phys. Lett. B365, 1(1996), and P. Braun-Munzinger, I. Heppe, and J. Stachel, Phys. Lett. B465, 15(1999).

[12] F. Becattini, M. Gazdzicki, and J. Sollfrank, Eur. Phys. J. C5, 143(1998).

[13] J. Cleymans and K. Redlich, Phys. Rev. Lett. 81, 5284(1998).

[14] P. Braun-Munzinger, D. Magestro, K. Redlich, and J. Stachel, Phys. Lett. B518, 41(2001).

[15] N. Xu and M. Kaneta, nucl-exp/0104022, April, 2001.

[16] K. Redlich, S. Hamieh, and A. Tounsi, J. Phys. G27, 413(2001).

[17] C.M. Ko, V. Koch, Z. Lin, K. Redlich, M. Stephanov, and X.N. Wang, Phys. Rev. Lett. 86, $5438(2001)$

[18] W. Schmitz et al. (NA45 Collaboration), these proceedings.

[19] T. Kolleger et al., (NA49 Collaboration), these proceedings.

[20] C. Quintans et al., (NA50 ollaboration), these proceedings.

[21] I. Bearden et al., (NA44 Collaboration), Phys. Rev. Lett 78, 2080(1997).

[22] E. Andersen et al., (WA97 collaboration), Phys. Lett. B433, 209(1998).

[23] V. Friese et al., (NA49 Collaboration), Quark Matter'01 Proceedings, Stony Brook, January, 2001.

[24] P. Bordalo et al., (NA50 Collaboration), Quark Matter'01 Proceedings, Stony Brook, January, 2001.

[25] M.C. Abreu et al., (NA50 Collaboration), Phys. Lett. B499, 85(2001).

[26] D. Teaney, J. Lauret, E. Shuryak, LANL Priprint, nucl-th/0104041, April, 2001.

[27] P. Huovinen, P. Kolb, U. Heinz, P.V. Ruuskanen, and S. Voloshin, Phys. Lett. B503, 58(2001).

[28] J. Schwinger, Phys. Rev. 82, 664(1951). 
[29] S. Soff, S. Bass, M. Bleicher, L. Bravina, M. Gorenstein, E. Zabrodin, H. Stöcker, and W. Greiner, Phys. Lett. B471, 89(1999).

[30] M. Bleicher, W. Greiner, H. Stöcker, and N. Xu, Phys. Rev. C62, R1901(2000).

[31] S. Johnson, B. Jacak, and A. Drees, E. J. Phys. C, in print, (2001).

[32] S. Soff et al., J. Phys. G: Nucl. Part. 27, 449(2001).

[33] P. Filip and E. Kolomeitsev, these proceedings; Phys. Rev. C64, 504905(2001).

[34] D. Hofman et al., (E917 Collaboration) these proceedings.

[35] S. Gavin and M. Gyulassy, Phys. Lett. B214, 241(1988).

[36] J. Hüfner, Y. Kurihara, and H. Pirner, Phys. Lett. B215, 218(1988).

[37] J. Blaizot and J. Ollitrault, Phys. Lett. B217, 392(1989).

[38] B. Anderson, G. Gustafson, G. Ingelman, and T. Sjöstrand, Phys. Rep. 97, 31(1983).

[39] P. Braun-Munzinger and J. Stachel, Phys. Lett. B490, 196(2000)

[40] R. Thews, M. Schroedter and J. Rafelski, J. Phys. G27, 715(2001); R. Thews, M. Schroedter and J. Rafelski, LANL Preprint hep-ph/0007323 v3, Feb. (2001).

[41] H. Sorge, Phys. Lett. B402, 251(1997).

[42] J.-Y. Ollitrault, Phys. Rev. D46, 229(1992).

[43] B. Zhang, M. Gyulassy, and C.M. Co, Phys. Lett. B455, 45(1999).

[44] M. Gyulassy, I. Vitev and X.N. Wang, Phys. Rev. Lett. 86, 2537(2001).

[45] D. Teaney, J. Lauret, and E.V. Shuryak, Phys. Rev. Lett. 86, 4783(2001).

[46] Z.W. Lin and C.M. Ko, LANL Preprint, nucl-th/0108039.

[47] L. Bravina, L.P. Csernai, A. Faessler, C. Fuchs, E.E. Zabrodin, nucl-th/0107056, July, 2001.

[48] P. Sorensen et al. (STAR Collaboration), these proceedings.

[49] C. Adler et al., (STAR Collaboration), Phys. Rev. Lett., 87, 182301(2001).

[50] P. Huovinen, P.F. Kolb, U. Heinz, P.V. Ruuskanen, and S. Voloshin, Phys. Lett. B503, $58(2001)$. 